\title{
Chronic ephedrine administration decreases brown adipose tissue activity in a randomised controlled human trial: implications for obesity
}

\author{
Andrew L. Carey • Renata Pajtak • Melissa F. Formosa • Bruce Van Every • \\ David A. Bertovic • Mitchell J. Anderson • Nina Eikelis • Gavin W. Lambert • \\ Victor Kalff • Stephen J. Duffy • Martin H. Cherk • Bronwyn A. Kingwell
}

Received: 21 December 2014 / Accepted: 5 February 2015 /Published online: 1 March 2015

(C) Springer-Verlag Berlin Heidelberg 2015

\begin{abstract}
Aims/hypothesis Brown adipose tissue (BAT) activation increases energy expenditure and may have therapeutic potential to combat obesity. The primary activating and adaptive signal for BAT is via $\beta$-adrenergic signalling. We previously demonstrated that human BAT is acutely responsive to oral administration of the sympathomimetic, ephedrine. Here we aimed to determine whether adaptive thermogenesis can be induced via chronic treatment with ephedrine.

Methods Twenty-three healthy young men, recruited from the general public in Melbourne, Australia, who were nonsmokers, physically inactive and non-medicated with no prior
\end{abstract}

Electronic supplementary material The online version of this article (doi:10.1007/s00125-015-3543-6) contains peer-reviewed but unedited supplementary material, which is available to authorised users.

A. L. Carey $(\bowtie) \cdot$ R. Pajtak · M. F. Formosa - D. A. Bertovic ·

M. J. Anderson · S. J. Duffy $\cdot$ B. A. Kingwell

Metabolic and Vascular Physiology Laboratory, Baker IDI Heart and

Diabetes Institute, PO Box 6492, St Kilda Road Central,

Melbourne, VIC 8008, Australia

e-mail: andrew.carey@bakeridi.edu.au

B. Van Every $\cdot$ V. Kalff $\cdot$ M. H. Cherk

Department of Nuclear Medicine, Alfred Hospital, Melbourne, VIC, Australia

D. A. Bertovic $\cdot$ S. J. Duffy $\cdot$ B. A. Kingwell

Department of Cardiology, Alfred Hospital, Melbourne, VIC, Australia

N. Eikelis $\cdot$ G. W. Lambert

Human Neurotransmitters Laboratory, Baker IDI Heart and Diabetes Institute, Melbourne, VIC, Australia

G. W. Lambert

Central Clinical School, Monash University, Melbourne, VIC, Australia history of cardiovascular disease or diabetes were recruited for this study. They were assigned to receive either $1.5 \mathrm{mg} \mathrm{kg}^{-1}$ day $^{-1}$ ephedrine ('active' group; $n=12$, age 23 \pm 1 years, BMI $\left.24 \pm 1 \mathrm{~kg} / \mathrm{m}^{2}\right)$ or placebo $(n=11 ; 22 \pm 2$ years, $23 \pm 2 \mathrm{~kg} / \mathrm{m}^{2}$ ) for 28 days in a randomised (computer-generated random order sequence), placebo-controlled, parallelgroup trial. Participants and all investigators were blinded to treatments. Body composition was measured before and after the intervention by dual energy X-ray absorptiometry. BAT activity, measured via ${ }^{18} \mathrm{~F}$-fluorodeoxyglucose positron emission tomography-computed tomography, in response to a single dose of $2.5 \mathrm{mg} / \mathrm{kg}$ ephedrine, was the primary outcome measure to be determined before and after the 28 day treatment period.

Results Twenty-eight individuals were randomised and consented to the study. Twenty-three completed the trial and only these participants were included in the final analyses. After 28 days of treatment, the active group lost a significant amount of total body fat (placebo $1.1 \pm 0.3 \mathrm{~kg}$, ephedrine $-0.9 \pm$ $0.5 \mathrm{~kg} ; p<0.01$ ) and visceral fat (placebo $6.4 \pm 19.1 \mathrm{~g}$, ephedrine $-134 \pm 43 \mathrm{~g} ; p<0.01$ ), with no change in lean mass or bone mineral content compared with the placebo group. In response to acute ephedrine, BAT activity (change in mean standardised uptake value: placebo $-3 \pm 7 \%$, ephedrine $-22 \pm 6 \%$ ) and the increase in systolic blood pressure were significantly reduced $(p<0.05)$ in the active group compared with placebo.

Conclusions/interpretation Chronic ephedrine treatment reduced body fat content, but this was not associated with an increase in BAT activity. Rather, chronic ephedrine suppressed BAT glucose disposal, suggesting that chronic ephedrine treatment decreased, rather than increased, BAT activity.

Trial registration: ClinicalTrials.gov NCT02236962

Funding: This study was funded by the National Health and Medical Research Council of Australia Program Grant 
(1036352) and the OIS scheme from the Victorian State Government.

Keywords Adaptive thermogenesis $\cdot$ Brown fat · Cold · Energy expenditure $\cdot$ Ephedrine $\cdot$ Noradrenaline $\cdot$ Norepinephrine - Sympathomimetic · Type 2 diabetes · Uncoupling protein $\cdot$ White fat

$\begin{array}{ll}\text { Abbreviations } & \\ \text { AR } & \text { Adrenergic receptor } \\ \text { BAT } & \text { Brown adipose tissue } \\ \text { DEXA } & \text { Dual energy X-ray absorptiometry } \\ \text { NA } & \text { Noradrenaline } \\ \text { PET-CT } & \begin{array}{l}\text { Positron emission tomography-computerised } \\ \text { tomography }\end{array} \\ \text { SUV } & \text { Standardised uptake value } \\ \text { UCP-1 } & \text { Uncoupling protein-1 } \\ \text { WAT } & \text { White adipose tissue }\end{array}$

\section{Introduction}

The conclusive identification of functional brown/beige adipose tissue (BAT) in adult humans [1-5] and its functional impairment in obesity $[3,6,7]$ has focussed attention on this tissue as an anti-obesity target. This impairment is due to the oxidative capacity of BAT, which, while relatively low and variable under basal conditions [8-10], can increase under conditions of chronic stimulation via a process called adaptive thermogenesis. Chronic cold exposure is the natural stimulus for adaptive thermogenesis, but mimicking cold-related signalling pathways also has the potential to elicit adaptive thermogenesis [8].

Cold-stimulated BAT adaptive thermogenesis in humans has recently been reported by four independent laboratories using different intermittent cold exposure protocols [11-13]. Prevailing views, therefore, suggested increasing cold stress by decreasing the ambient temperature in human dwellings and/or regular, purposeful 'cold-training' may reduce body weight, potentially via increasing BAT function and activity and decreasing body fat [14]. While there may be health benefits associated with regular intermittent cold exposure [15] it is unlikely to be effective for weight loss in humans. Prolonged cold exposure, regardless of intensity, will be difficult to sustain and, due to cold-stimulated hyperphagia, is unlikely to result in sustained weight loss and may actually promote weight gain $[16,17]$. For the purpose of reversing obesity, identifying pharmacological agents that increase BAT function without concomitant central hyperphagic signals would be most effective [8].

Recent pharmacological (thyroxine) [18] and nutritional (capsinoid extract) [13] interventions have provided indirect evidence that BAT function may be enhanced by chronic treatment with orally bioavailable agents. Pharmacological studies in humans investigating BAT thermogenesis to date have focussed primarily on sympathomimetic agents [6, 19-21]. These agents signal via adrenergic receptors (ARs), thereby notionally replicating both the facultative and adaptive thermogenic central sympathetic signal to BAT in response to cold exposure. We recently reported that high doses of the sympathomimetic ephedrine can activate BAT in some lean young men [6]. This is consistent with the effects of acute ephedrine in mice, a species where chronic administration of ephedrine is associated with adaptive thermogenesis. This chronic action of ephedrine in rodent BAT contrasts with other tissues where responsiveness to adrenergic agonists is reduced with chronic stimulation $[22,23]$. In this respect, ephedrine replicates some of the adaptive thermogenic effects of chronic cold exposure in rodents [24]. It is unknown, however, whether chronic treatment with sympathomimetic drugs can replicate coldstimulated adaptive thermogenesis in humans. While chronic use of these agents is not advised in humans due to significant cardiovascular side effects, they represent a suitable drug class for proof-of-concept studies. With the advent of positron emission tomography-computerised tomography (PET-CT) imaging to semi-quantitatively and directly measure BAT activity, it is now possible to investigate this question. Accordingly, the aim of the present study was to determine whether or not chronic treatment with the orally bioavailable sympathomimetic ephedrine increases BAT activity in response to a single dose of this drug.

\section{Methods}

Twenty-three young male participants (no history/clinical evidence of cardiovascular disease and either diabetes, impaired fasting glucose or impaired glucose tolerance, unmedicated, physically inactive and non-smokers) took part in this study. The study was approved by the Alfred Hospital Ethics Committee and performed in accordance with the Declaration of Helsinki, Sixth Revision, 2008. A Consolidated Standards of Reporting Trials (CONSORT) checklist and flow diagram is presented in electronic supplementary material (ESM) Fig. 1. All patients provided written informed consent.

Study design This study was a randomised, double-blinded, placebo-controlled trial. Participants were randomised into two groups to receive either active treatment (ephedrine $1.5 \mathrm{mg} \mathrm{kg}^{-1} \mathrm{day}^{-1}$ ) or placebo (lactose). Dosing was selected based on pilot studies that determined $1.5 \mathrm{mg} \mathrm{kg}^{-1}$ day $^{-1}$ to be the highest single dose that could be safely taken while unsupervised outside the laboratory. Sample sizes were based on power calculations determined from our prior studies $[6,25$, 26]. Participants were randomised by The Alfred Hospital 
Clinical Trials Pharmacy staff in blocks of 4-6 using Microsoft Excel (v2007) to generate a random order sequence.

Participants visited the laboratory three times, all located within the Departments of Cardiology and Nuclear Medicine at The Alfred Hospital. On visit 1, after obtaining informed consent a medical screen and body composition analysis (dual energy X-ray absorptiometry [DEXA]) were conducted. Visits 2 and 3 occurred before and after the treatment intervention, respectively, and included assessment of BAT activity (PET$\mathrm{CT}$ ), whole-body energy expenditure (indirect calorimetry) and blood variables (blood glucose, NEFA and noradrenaline [NA; norepinephrine]) in response to a single dose of ephedrine $\left(2.5 \mathrm{mg} \mathrm{kg}^{-1}\right)$. These measurements were made using a protocol previously established by us [6]. The day after visit 2 , participants began taking a single oral dose of ephedrine $\left(1.5 \mathrm{mg} \mathrm{kg}^{-1}\right)$ or placebo between 09:00 and 11:00 hours daily for 28 days. Within 2 days of taking their final dose, participants returned to the laboratory (visit 3 ) and their body composition was reassessed via DEXA.

Previous studies indicate BAT activity varies seasonally $[2$, 27]. While we conducted experiments between March and October, participants were randomised in blocks of four to six to minimise the potential for seasonal variation between treatment groups. We did not observe any variation in ephedrine-stimulated BAT activity between groups during pre-intervention experimental trials (visit 2).

Outcome measures The primary outcome measure was change in BAT activity. Secondary outcome measures comprised changes in basal and ephedrine-stimulated energy expenditure, body composition, circulating hormones, lipids and other metabolites.

\section{Experimental protocol}

Screening Initial screening involved clinical history and examination by a physician and measurement of physical characteristics, including height, weight, waist:hip ratio, brachial artery blood pressure and 12-lead ECG. A fasting blood sample was drawn for measurement of lipid profile (total, LDLand HDL-cholesterol and triacylglycerol), insulin, $\mathrm{NA}, \mathrm{HbA}_{1 \mathrm{c}}$ and glucose. An OGTT was then performed. Briefly, participants consumed a $75 \mathrm{~g}$ glucose solution, after which blood glucose was measured at 60 and $120 \mathrm{~min}$. Body composition (lean, bone and fat mass) was measured using DEXA.

BAT activation trials Before and within 24-48 $\mathrm{h}$ after the 4 week drug intervention, participants were given a standardised meal $(3,180 \mathrm{~kJ} ; 84 \%$ carbohydrate, $13 \%$ protein, $3 \%$ fat) to consume the evening prior to attending the laboratory (at 18:00-22:00 hours) on both experimental days.
Laboratory temperature was $20-22^{\circ} \mathrm{C}$. Upon arrival at 07:30-08:00 hours after an overnight fast and having abstained from vigorous exercise, caffeine, smoking and alcohol consumption for at least 2 days prior, participants voided and changed into standard hospital scrubs and socks. They then consumed a telemetric pill for recording of core temperature (Cortemp, HQ Inc, Palmetto, FL, USA) and a venous cannula was inserted into an antecubital vein. Brachial blood pressure (Philips Suresigns VS3; Philips Medical Systems, Andover, MA, USA) was measured every $15 \mathrm{~min}$ and heart rate (Cortemp) was continuously recorded. Participants then rested in a supine position for $2 \mathrm{~h}$ while covered with two blankets to ensure thermoneutrality.

After resting, energy expenditure was measured via indirect calorimetry, a blood sample was taken and participants then consumed $2.5 \mathrm{mg} \mathrm{kg}^{-1}$ ephedrine hydrochloride in a gelatine capsule with water. Blood samples were taken at 15 , 30, 60 and $90 \mathrm{~min}$ after drug ingestion for subsequent analyses (described below). Participants were injected with an FDG tracer for BAT glucose uptake assessment via PET-CT $60 \mathrm{~min}$ after drug ingestion. Energy expenditure was again measured via indirect calorimetry $60-90 \mathrm{~min}$ after drug ingestion because this time was predicted to correspond to peak plasma NA concentrations and BAT activity $[6,19]$.

Indirect calorimetry Energy expenditure was measured with a ParvoMedics TrueOne 2400 metabolic analyser (ParvoMedics Inc, East Sandy, UT, USA). Mixed expired gases were measured after 10-min equilibration. Energy expenditure and respiratory exchange ratio were calculated and averaged over $20 \mathrm{~min}$ [6].

PET-CT imaging PET-CT imaging and analyses were conducted as previously described [6]. PET-CT variables resulted in an effective radiation dose of $<7 \mathrm{mSv}$ per scan, therefore the maximum radiation dose administered (including DEXA) was $14 \mathrm{mSv}$ per participant. PET-CT images were acquired and reconstructed using a Philips Gemini Dual PET-CT scanner (Philips, Andover, MA, USA). Scans were analysed using $4 \mathrm{~mm}$ thick coronal slices on an Extended Brilliance Workstation (Philips).

Analysis focussed on the supraclavicular adipose tissue depot as this area has been consistently shown in numerous studies to demonstrate activity and/or molecular markers of BAT in humans $[1-7,9,12,13,20,28-30]$. Recent evidence also suggests that in humans this tissue is likely to be predominately beige adipose tissue [29, 31-33]. For simplicity, hereafter this tissue will be referred to as BAT and activity in this region will be considered to represent 'BAT activity'. Tissue CT radiodensity in the established range of -180 to -10 Hounsfield units within this region was considered to represent adipose tissue $[6,20,21,34]$. 
We quantified maximum standardised uptake value (SUVmax) in supraclavicular adipose (representative of BAT) and subcutaneous adipose (upper arm, representative of white adipose tissue [WAT]). Basal SUVmax of supraclavicular adipose is $\sim 0.8-1.0$, which is $\geq 2$ standard deviations above that of subcutaneous WAT $(\sim 0.4)$ [6]. In order to better represent total activity in this region we also conducted volumetric analyses to encompass a larger tissue region. We did not, however, observe increased supraclavicular adipose SUVmax above basal levels in the majority of participants in response to acute ephedrine. In light of this finding, and given the prolonged interventional nature of this study, we conducted a 'fixed volume' analysis [35]. From three consecutive PET image slices (4 mm thickness) within the supraclavicular region, SUVmean from regions of interest of $100 \mathrm{~mm}^{2}$ were determined on left and right sides. These data are, therefore, reported as SUVmean per 2, $400 \mathrm{~mm}^{3}$. SUVmax and SUVmean are determined in units of $\mathrm{g} / \mathrm{ml}$, however neither our method of reconstruction and data analysis nor the formula to quantify these variables are quantitative. Data are, therefore, presented simply as 'SUVmax' and 'SUVmean' without units.

Biochemical analyses Where required, plasma was centrifuged and frozen for analyses. Plasma was measured for glucose, total cholesterol, HDL-cholesterol, LDL-cholesterol, triacylglycerol, insulin, $\mathrm{HbA}_{1 \mathrm{c}}$ and NA as described [6]. Plasma NEFA levels were measured using a commercially available kit (Waco Diagnostics, Richmond, VA, USA).

Statistical analyses Physical characteristics between groups and change in body composition between groups as a result of treatments were compared using unpaired two-tailed Student's $t$ tests. To determine effects of acute ephedrine treatment on energy expenditure, the change in response to the acute ephedrine treatment was determined both before (Pre) and after (Post) the chronic treatment in each group. The change in this value from Pre to Post was then determined and this change was compared between the two treatment groups using an unpaired two-tailed Student's $t$ test. The same analysis was applied for comparison of haemodynamic, core temperature and circulating factor data, however the change in response to acute ephedrine treatment for each group and time-point was determined by subtracting the basal value from the mean of values obtained between $60-90 \mathrm{~min}$ after the acute ephedrine dose. As previously reported by us [6] and others [19], the peak in acute physiological responses to oral ephedrine varies between individuals but occurs within this period.

Effects on BAT activity were determined as described above for body composition data, however since PET-CT data were not normally distributed, a non-parametric Mann-Whitney $U$ test was used to compare between groups. Analyses were conducted using SPSS (v15) and Microsoft Excel.
Results are expressed as mean $\pm \mathrm{SEM}$, and results were considered significant when $p \leq 0.05$.

\section{Results}

Baseline participant characteristics are presented in Table 1. Groups were not statistically different for all criteria. Table 2 shows body composition at baseline and in response to the 28day intervention. The change in total mass (change placebo 1.1 $\pm 0.3 \mathrm{~kg}$; ephedrine $-0.9 \pm 0.5 \mathrm{~kg}$ ), body fat mass (change placebo $0.5 \pm 0.2 \mathrm{~kg}$; ephedrine $-1.1 \pm 0.3 \mathrm{~kg}$ ), per cent body fat (change placebo $0.4 \pm 0.2 \%$; ephedrine $-1.2 \pm 0.3 \%$ ) and estimated visceral adipose tissue mass (change placebo $6.4 \pm$ $19.1 \mathrm{~g}$; ephedrine $-134 \pm 43 \mathrm{~g}$ ) between groups were all significantly reduced after chronic ephedrine treatment only (Table 2; $p<0.01)$. Lean mass and bone mineral content were unchanged.

BAT activity after chronic treatment was significantly lower in response to acute ephedrine in the chronic ephedrine

Table 1 Participant baseline characteristics

\begin{tabular}{|c|c|c|c|}
\hline Patient characteristic & Placebo & Ephedrine & $p$ values \\
\hline$n$ & 11 & 12 & \\
\hline \multicolumn{4}{|l|}{ Physical characteristics } \\
\hline Age (years) & $22 \pm 2$ & $23 \pm 1$ & 0.44 \\
\hline Height (cm) & $178 \pm 5$ & $178 \pm 2$ & 0.89 \\
\hline Weight (kg) & $74 \pm 2$ & $80 \pm 3$ & 0.13 \\
\hline BMI $\left(\mathrm{kg} / \mathrm{m}^{2}\right)$ & $23 \pm 2$ & $25 \pm 1$ & 0.11 \\
\hline Body fat (\%) & $23 \pm 3$ & $26 \pm 2$ & 0.45 \\
\hline Waist:hip & $0.88 \pm 0.39$ & $0.88 \pm 0.20$ & 0.92 \\
\hline \multicolumn{4}{|l|}{ Resting cardiovascular variables } \\
\hline Systolic blood pressure (mmHg) & $120 \pm 5$ & $118 \pm 2$ & 0.67 \\
\hline Diastolic blood pressure (mmHg) & $74 \pm 4$ & $73 \pm 2$ & 0.61 \\
\hline Heart rate (bpm) & $63 \pm 6$ & $67 \pm 2$ & 0.33 \\
\hline \multicolumn{4}{|c|}{ Fasting plasma hormones, metabolites and lipids } \\
\hline NA (pmol/1) & $936 \pm 134$ & $975 \pm 180$ & 0.56 \\
\hline Insulin (pmol/l) & $49 \pm 17$ & $72 \pm 31$ & 0.50 \\
\hline Glucose (mmol/l) & $4.9 \pm 0.2$ & $5.0 \pm 0.1$ & 0.43 \\
\hline $\mathrm{HbA}_{1 \mathrm{c}}(\%)$ & $5.3 \pm 0.2$ & $5.2 \pm 0.1$ & 0.59 \\
\hline $\mathrm{HbA}_{1 \mathrm{c}}(\mathrm{mmol} / \mathrm{mol})$ & $34 \pm 2$ & $34 \pm 1$ & 0.59 \\
\hline Total cholesterol (mmol/l) & $4.4 \pm 0.8$ & $4.1 \pm 0.2$ & 0.37 \\
\hline HDL-cholesterol (mmol/l) & $1.1 \pm 0.1$ & $1.2 \pm 0.1$ & 0.22 \\
\hline LDL-cholesterol (mmol/l) & $2.7 \pm 0.6$ & $2.4 \pm 0.2$ & 0.32 \\
\hline Triacylglycerol (mmol/l) & $1.4 \pm 0.7$ & $1.1 \pm 0.1$ & 0.36 \\
\hline NEFA $(\mu \mathrm{mol} / 1)$ & $621 \pm 54$ & $533 \pm 30$ & 0.18 \\
\hline \multicolumn{4}{|l|}{ OGTT } \\
\hline 60 min glucose $(\mathrm{mmol} / \mathrm{l})$ & $6.0 \pm 1.3$ & $6.3 \pm 0.5$ & 0.64 \\
\hline 120 min glucose $(\mathrm{mmol} / \mathrm{l})$ & $4.9 \pm 0.7$ & $5.3 \pm 0.3$ & 0.39 \\
\hline
\end{tabular}

Values are mean \pm SEM

$p>0.05$ for all (groups compared using an unpaired Student's $t$ test) 
Table 2 Body composition

\begin{tabular}{|c|c|c|c|c|c|c|}
\hline \multirow[t]{2}{*}{ Characteristic } & \multicolumn{3}{|l|}{ Placebo } & \multicolumn{3}{|l|}{ Ephedrine } \\
\hline & Pre & Post & Change & Pre & Post & Change \\
\hline Total mass (kg) & $74.3 \pm 2.2$ & $75.4 \pm 2.3$ & $1.1 \pm 0.3$ & $80.0 \pm 2.8$ & $79.1 \pm 2.9$ & $-0.9 \pm 0.5^{\mathrm{a}}$ \\
\hline Lean mass (kg) & $54.6 \pm 1.8$ & $55.1 \pm 1.9$ & $0.56 \pm 0.3$ & $55.9 \pm 1.3$ & $56.2 \pm 1.4$ & $0.24 \pm 0.3$ \\
\hline Fat mass $(\mathrm{kg})$ & $16.8 \pm 2.1$ & $17.3 \pm 2.1$ & $0.50 \pm 0.2$ & $20.9 \pm 2.3$ & $19.8 \pm 2.3$ & $-1.1 \pm 0.3^{\mathrm{a}}$ \\
\hline Fat mass ( $\%$ total mass) & $23.2 \pm 2.6$ & $23.6 \pm 2.5$ & $0.4 \pm 0.2$ & $26.6 \pm 2.2$ & $25.4 \pm 2.2$ & $-1.2 \pm 0.3^{\mathrm{a}}$ \\
\hline $\operatorname{eVAT}(g)$ & $493 \pm 109$ & $500 \pm 116$ & $6.4 \pm 19.1$ & $512 \pm 73$ & $378 \pm 95$ & $-134 \pm 43^{\mathrm{a}}$ \\
\hline Bone mineral content $(\mathrm{g})$ & $2,915 \pm 95$ & $2,917 \pm 95$ & $1.6 \pm 94$ & $3,150 \pm 94$ & $3,132 \pm 94$ & $-18 \pm 8$ \\
\hline
\end{tabular}

Values are mean \pm SEM

${ }^{a} p<0.01$ for change between groups, ephedrine treatment significantly different compared with placebo (the pre-post change was compared between treatment groups using an unpaired Student's $t$ test)

eVAT, estimated visceral adipose tissue

treated group but was unchanged in the placebo group when measured as change in SUVmax (Fig. 1a; mean change placebo $-3 \pm 6 \%$; ephedrine $-13 \pm 7 \%, p=0.03$ ) or SUVmean (Fig. 1b; mean change placebo $-3 \pm 7 \%$; ephedrine $-22 \pm 6 \%$; $p=0.01)$. SUVmax in WAT was half that of BAT and was unchanged in both groups in response to acute ephedrine before chronic treatment (placebo pre $0.48 \pm 0.02$, placebo post $0.45 \pm 0.02$; ephedrine pre $0.45 \pm 0.02$, ephedrine post $0.43 \pm$ $0.03)$.

Basal energy expenditure did not change as a result of chronic ephedrine treatment (Table 3 ). The increase in energy expenditure in response to acute ephedrine was unchanged after the chronic treatment period between groups (Table 3). The respiratory exchange ratio was not affected by either acute or chronic ephedrine treatment (Table 3).

The change in systolic blood pressure, heart rate, core temperature, blood glucose, plasma NA and plasma NEFAs in response to the acute dose of ephedrine are shown in Fig. 2 both before (Pre) and after (Post) the 28-day chronic ephedrine

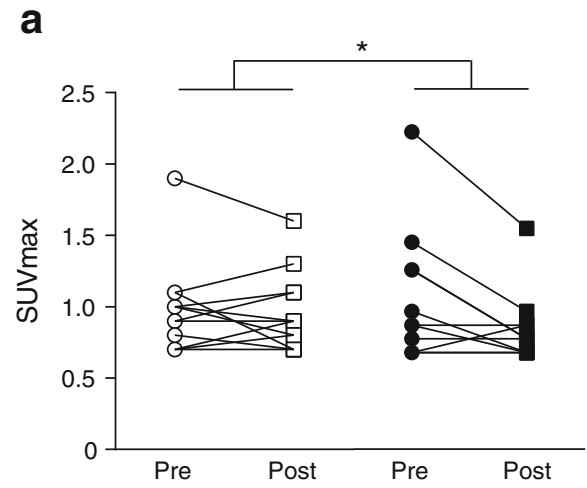

Fig. 1 (a) SUVmax and (b) SUVmean values plotted for each individual in supraclavicular brown/beige adipose tissue in response to treatment with a single dose of ephedrine $\left(2.5 \mathrm{mg} \mathrm{kg}^{-1}\right)$, before (pre) and after (post) 28 days treatment with placebo or ephedrine $\left(1.5 \mathrm{mg} \mathrm{kg}^{-1}\right.$ day $\left.^{-1}\right)$. SUVmax group mean values: placebo pre (open circles) $0.98 \pm 0.09$; post (open squares) $0.96 \pm 0.08$; ephedrine pre (closed circles) $0.98 \pm 0.13$; post intervention. Compared with placebo, in the chronic ephedrine treatment group the change from pre- to post-intervention was significantly less for systolic blood pressure $(p<0.05)$ and blood glucose $(p<0.001)$, and trended towards a reduction for plasma NA $(p=0.06)$. There was no difference in the response between groups for heart rate, core temperature and NEFA.

\section{Discussion}

In the present study, we hypothesised that chronic treatment with ephedrine would induce adaptive thermogenesis in BAT in adult humans, resulting in increased BAT activity. Contrary to this hypothesis, BAT activity was significantly reduced by 28 days of ephedrine treatment. This effect is likely to be due to ephedrine tolerance and may have implications for conditions of chronic sympathetic activation. Thus, chronic stress, hypertension and established obesity have all been associated

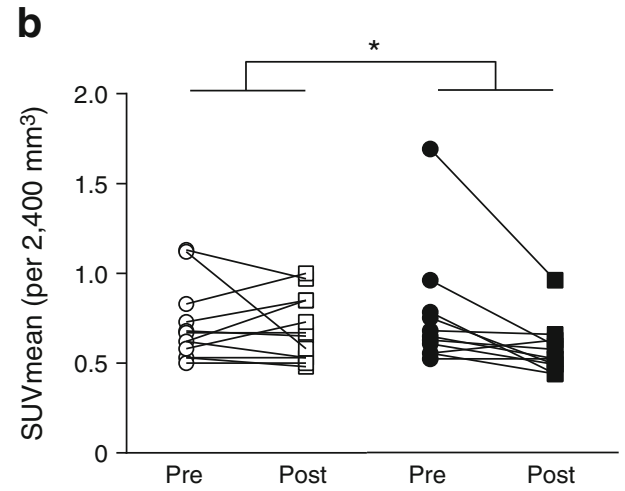

(closed squares) $0.80 \pm 0.07$. SUVmean group mean values: placebo pre $0.71 \pm 0.06$; post $0.70 \pm 0.05$; ephedrine pre $0.76 \pm 0.10$; post $0.55 \pm 0.04$. $* p<0.05$ for change from pre- to post-treatment between groups (the prepost change was compared between treatment groups using a non-parametric Mann-Whitney $U$ test) 
Table 3 Indirect calorimetry

\begin{tabular}{|c|c|c|c|c|c|c|}
\hline \multirow[t]{2}{*}{ Characteristic } & \multicolumn{3}{|l|}{ Placebo } & \multicolumn{3}{|c|}{ Chronic ephedrine } \\
\hline & Pre & Post & Change & Pre & Post & Change \\
\hline Basal energy expenditure $\left(\mathrm{kJ} \mathrm{h}^{-1}\right)$ & $287 \pm 17$ & $285 \pm 8$ & $-2 \pm 14$ & $286 \pm 16$ & $287 \pm 10$ & $0.4 \pm 15$ \\
\hline Ephedrine-stimulated energy expenditure $\left(\mathrm{kJ} \mathrm{h}^{-1}\right)$ & $326 \pm 14$ & $322 \pm 10$ & $-4 \pm 13$ & $340 \pm 11$ & $323 \pm 11$ & $-16 \pm 11$ \\
\hline Basal RER & $0.84 \pm 0.02$ & $0.82 \pm 0.01$ & $-0.02 \pm 0.02$ & $0.84 \pm 0.02$ & $0.81 \pm 0.01$ & $-0.03 \pm 0.01$ \\
\hline Acute ephedrine RER & $0.82 \pm 0.01$ & $0.82 \pm 0.01$ & $0.01 \pm 0.02$ & $0.85 \pm 0.04$ & $0.84 \pm 0.02$ & $-0.02 \pm 0.04$ \\
\hline
\end{tabular}

Whole-body energy expenditure and RER measured before (basal) and 70-90 min after (ephedrine-stimulated) ingestion of a single dose of ephedrine $\left(2.5 \mathrm{mg} \mathrm{kg}^{-1}\right)$, measured before (pre) and after (post) 28 days treatment with either placebo or ephedrine $\left(1.5 \mathrm{mg} \mathrm{kg}^{-1}\right.$ day $\left.^{-1}\right)$. Change represents the difference between pre and post

Values are mean \pm SEM. (The pre-post change was compared between treatment groups using an unpaired Student's $t$ test)

RER, respiratory exchange ratio

with sympathetic activation, which may lead to blunted sympathetic responsiveness in certain tissues [36]. Our findings may, therefore, contribute to an explanation for the observed reduction in BAT function in obese individuals.
BAT biopsies and other measures were not possible to directly measure BAT function or BAT sympathetic nerve activity. Nevertheless, a reduction in the increase in systolic blood pressure and blood glucose, and a trend towards a blunting of
Fig. 2 Changes $(\Delta)$ in (a) systolic blood pressure (SBP), (b) heart rate (HR), (c) core temperature, (d) blood glucose, (e) plasma NA ( $p=0.06$ for change from pre- to posttreatment between groups) and (f) NEFA from immediately prior to ingestion of a single dose of ephedrine $(2.5 \mathrm{mg} / \mathrm{kg})$ to the mean of values taken between 60 and $90 \mathrm{~min}$ after ingestion of the dose of ephedrine, before (pre) and after (post) 28 days treatment with placebo or ephedrine $\left(1.5 \mathrm{mg} \mathrm{kg}^{-1} \mathrm{day}^{-1}\right)$. Open bars, placebo; closed bars, ephedrine. ${ }^{*} p<0.05$ for the change from preto post-treatment between groups, $* * p<0.001$ for change from preto post-treatment between groups (the pre-post change was compared between treatment groups using an unpaired Student's $t$ test)
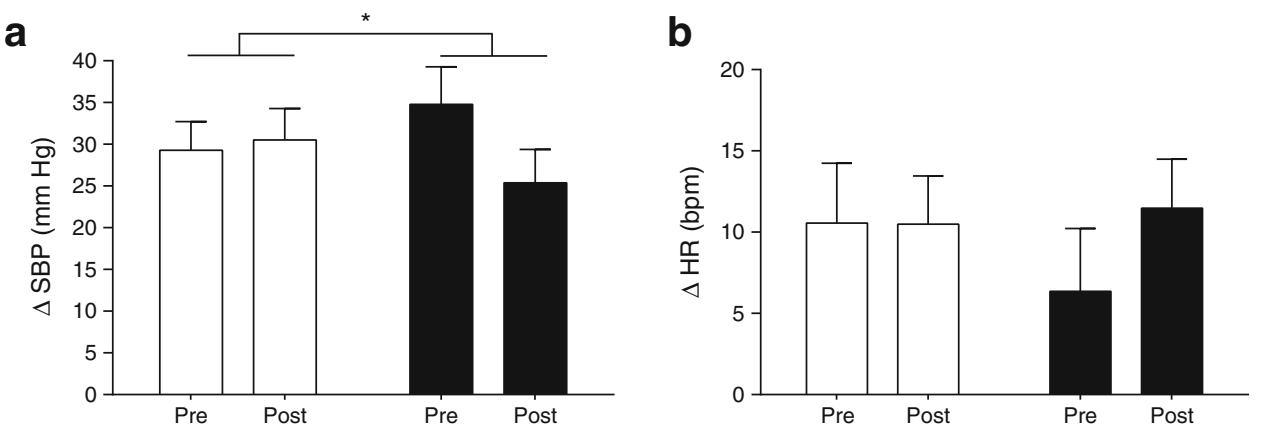

C
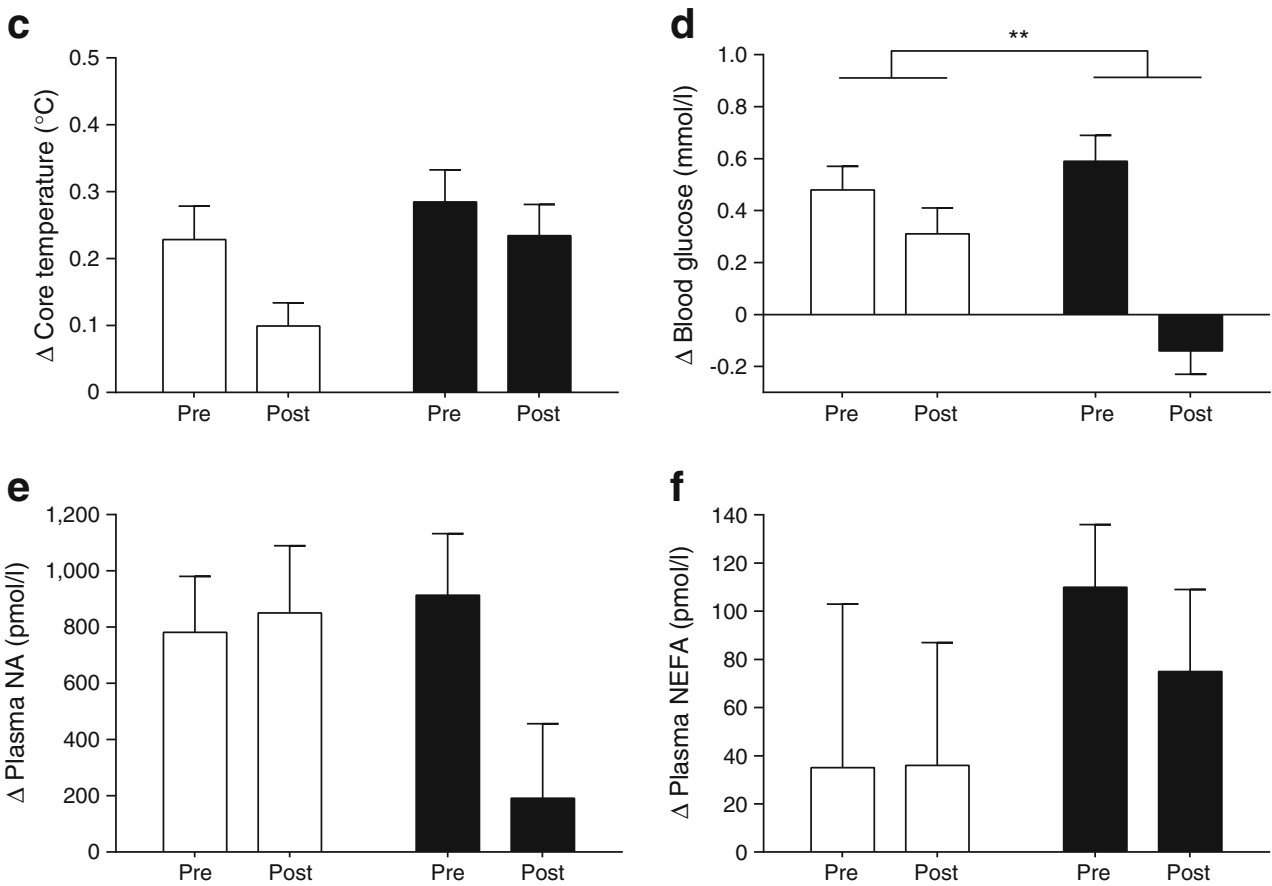
the rise in plasma NA in response to acute ephedrine in the chronic ephedrine treatment group support a blunting of sympathetic responsiveness to this protocol. The sympathomimetic action of ephedrine is based on increased endogenous NA release from sympathetic nerve terminals and blockade of its reuptake, thereby increasing and prolonging synaptic NA concentrations, spillover into circulation and subsequent exposure to cells [37]. Thus, the mechanism by which chronic ephedrine treatment downregulates adipose adrenergic sensitivity to acute ephedrine in humans is likely to be due to direct downregulation of $\beta$-AR on target tissues and/or altered synaptic NA release/reuptake via synaptic NA transporters. Blunting the rise in plasma NA response to acute ephedrine in the chronic ephedrine group indicates an alteration in the synaptic regulation of NA release and/ or reuptake. Further, in rodents, chronic $\beta A R$ stimulation with isoproterenol decreases $\beta$-AR sensitivity and receptor density [38], therefore both mechanisms are probably involved.

In many tissues, stimulation of $\beta$-ARs results in both shortterm (hours) and chronic (days) downregulation of receptor sensitivity and density, respectively [23, 39]. Rodent studies, however, indicate that BAT $\beta 3$-AR are unaffected, thus allowing adaptive thermogenesis to occur in response to persistent stimulation [22, 23]. Accordingly, chronic ephedrine treatment increases uncoupling protein-1 (UCP-1) and BAT activity in response to acute ephedrine treatment in mice [24], and similar results have been reported with $\beta 3-\mathrm{AR}$ agonist treatment in adult dogs, which reportedly have functionally similar BAT to adult humans [40]. It is unknown which $\beta$ ARs are primarily responsible for human BAT thermogenesis, however, while expressed [4], $\beta 3$-AR content is likely to be low [23] and to have little [41, 42] or no [43] involvement in human thermogenesis. The present data, therefore, support the notion that human BAT is functionally distinct from that of small rodents and other larger mammals such as dogs. Human BAT has recently been reported to be composed predominately of beige rather than classic brown adipocytes [29, 31-33], and a recent in vitro study highlighted that human and mouse BAT cells express UCP-1 in an opposing manner in response to all-trans retinoic acid treatment [44]. Moreover, chronic ephedrine treatment mimics adaptive thermogenesis in mice [24] and no study has reported a reduction in facultative or adaptive thermogenesis in classic brown or beige adipose depots in mice in response to sympathetic stimuli. Therefore, it is likely human BAT requires alternate stimuli to become responsive to adrenergic/sympathetic inputs, and this may contribute to the failure of $\beta 3$-AR agonists to increase BAT activity in humans [45].

Mice require all $\beta$-ARs for full function of classic BAT, but are predominantly dependant on the $\beta 3$-AR $[22,23,46]$. Since human BAT is not generally as well adapted as murine BAT it may require proliferative and adipogenic stimulation to precede browning to maximise adaptive thermogenesis. These processes may be more dependent on $\beta 1-A R$ than $\beta 3-A R$ signalling [23, 47], thus potentially explaining a desensitisation rather than adaptive phenomenon more representative of tissues that highly express $\beta 1 / 2$-ARs. From an obesity therapeutic perspective, in future it would, therefore, be prudent to consider human BAT as genotypically and phenotypically distinct from murine BAT. Additionally, altered sympathetic function has been implicated as both a cause and consequence of obesity [36] and weight loss reverses dysregulated basal and glucose-stimulated sympathetic responsiveness [48, 49]. Neither reduced BAT activity nor sympathetic over-activity have been causatively linked to obesity. Since, however, BAT activity is reduced and basal sympathetic nervous system activity is increased in obesity, the present data connecting chronic sympathetic over-stimulation to reduced BAT activity strengthens the hypothesis that these observations are linked.

In our previous study, upper thoracic BAT activity, measured as SUVmax, in response to acute ephedrine was $\sim 2.0$, which was $\sim 1.0$ higher than the acute response to placebo [6]. In the current study, baseline values for SUVmax in response to acute ephedrine were lower those of our previous study at $\sim 1.0$. Nevertheless, chronic ephedrine treatment reduced SUVmax compared with placebo. Considering that SUVmax of deep upper thoracic BAT is significantly higher than subcutaneous WAT depots $(\sim 100 \%$ and $>2$ SDs in the present study and our previously reported basal levels [6]), it is clearly distinct in having higher metabolic activity. Accordingly, human upper thoracic BAT is now characterised as a distinct brown/beige fat depot and, therefore, may fluctuate in oxidative capacity. Nevertheless, this absolute level of BAT activity contributes only a small portion of whole-body energy expenditure [8]. While statistically significant, the small reduction observed here would be unlikely to contribute meaningfully to whole-body energy expenditure under the present experimental conditions. However, other physiological stimuli that activate BAT may result in greater differences of larger absolute magnitude, therefore it is not yet possible to conclusively evaluate the physiological relevance of these findings. Conversely, since we did not observe an increase in activity above a predetermined basal level, measurement of basal BAT activity before and after the present intervention would have been of limited value. Nevertheless, while ethical considerations related to radiation exposure prevented additional basal PETCT scans in the current study, future intervention studies should aim to study the functional relevance of BAT under unstimulated conditions. The low proportion of participants for whom we observed acute ephedrine-stimulated activity approaching that of our previous study [6] could simply be related to only the smallest and/or leanest individuals being responsive; the lean group in our prior study (BMI $21 \mathrm{~kg} / \mathrm{m}^{2}$, body mass $66 \mathrm{~kg}$, body fat content $17 \%$ ) was significantly 
lighter with lower body fat content than that of the present cohort $\left(\sim 24 \mathrm{~kg} / \mathrm{m}^{2}, 76 \mathrm{~kg}, 25 \%\right)$.

Chronic ephedrine treatment resulted in a significant loss of body fat, particularly in the visceral compartment, with no loss of lean mass or bone mineral content. This highly desirable outcome was not attributable to any alteration in thermogenic function since neither basal nor ephedrine-stimulated energy expenditure changed in response to treatment. The likely explanation for the loss of body mass and fat is an increase in energy expenditure in combination with decreased energy intake. Ephedrine has a systemic half-life of $\sim 4 \mathrm{~h}$ and single ephedrine doses of 1-2.5 $\mathrm{mg} \mathrm{kg}^{-1}$ increase energy expenditure by $\sim 10-15 \%$ for several hours post-treatment, putatively mainly via increased muscle thermogenesis [19]. Based on the present data indicating no change in basal or ephedrinestimulated energy expenditure in response to chronic ephedrine treatment, tolerance does not develop with respect to the whole-body thermogenic response. Such shifts in energy balance may be compensated by increased gut and central orexigenic signals and, therefore, energy intake [50]; however, ephedrine is also an appetite suppressant [41]. We can only speculate as to the major contributing factor since body composition was not a primary outcome measure and food diaries (which are not particularly reliable) were not recorded.

While acute treatment with $2.5 \mathrm{mg} \mathrm{kg}^{-1}$ ephedrine did not result in increased BAT activity in the majority of participants in this study, and since we previously reported this dose would likely be the minimum necessary to activate BAT in lean adults [6], ideally we would have administered at least this dose daily in the present study. Due to the cardiovascular activation induced by this high dose, however, we opted to treat with a lower dose, hypothesising that the necessary adaptive adrenergic signalling events would occur in fat tissue whether or not significant thermogenesis was induced. Regardless, based on our observations, a higher dose would likely have only resulted in greater suppression of basal BAT FDG uptake. It is worth considering that the response to cold exposure after the chronic ephedrine intervention may differ to that of acute high-dose ephedrine. Cold exposure will activate BAT to a greater extent via physiological pathways that may result in differing signalling and/or substrate preference. This possibility remains to be investigated.

BAT function measured via FDG PET poses a number of technical limitations, including assessment of glucose uptake when lipids may be the predominant BAT substrate for thermogenesis. It is conceivable that in our studies of acute highdose ephedrine treatment glucose is not a major BAT substrate. This is, however, unlikely, since chronic cold exposure studies report similar results whether using PET imaging with FDG $[12,13]$ or an acetate tracer to measure whole tissue metabolism [11]. Nevertheless, this possibility also remains to be studied via a technique that assesses whole tissue energy expenditure.
In the present study, we provide evidence that, contrary to our hypothesis and in contrast to mice, chronic treatment with a sympathomimetic decreases basal BAT activity and likely thermogenic responsiveness to adrenergic stimuli. This finding represents an important difference between mouse and human BAT and the differing function of human BAT should be carefully considered when assessing future BAT-dependant therapeutic targets for obesity. Importantly, while reduced BAT activity has yet to be causatively linked to obesity, our data suggest that the elevated sympathetic activity observed in obesity may perpetuate weight gain by further reducing BAT function.

Acknowledgements The authors thank C. Despott (Department of Nuclear Medicine, Alfred Hospital, Melbourne, VIC, Australia) and S. Phillips (Human Neurotransmitters Laboratory, Baker IDI Heart and Diabetes Institute, Melbourne, VIC, Australia) for technical assistance, and the research participants for their time and interest in our study.

Funding This study was supported by a National Health and Medical Research Council of Australia Program Grant (1036352) and the OIS scheme from the Victorian State Government. BAK holds a National Health and Medical Research Council Senior Principal Research Fellowship (1059454). GWL holds a National Health and Medical Research Council Senior Principal Research Fellowship (1042492).

Contribution statement ALC, RP, MFF, BVE, DAB, MJA, VK, SJD, $\mathrm{MHC}$ and $\mathrm{BAK}$ were responsible for the conception and design of the study and experiments. ALC, RP, MFF, BVE, DAB, MJA, NE, GWL, VK, SJD, MHC and BAK acquired the data. ALC, RP, MFF, BVE, NE, GWL, VK, SJD, MHC and BAK analysed and interpreted the data. ALC, RP, MFF, BVE, DAB, MJA, NE, GWL, VK, SJD, MHC and BAK drafted and critically revised the manuscript. All authors approved this version of the manuscript. ALC is the guarantor of this work.

Duality of interest statement The authors declare that there is no duality of interest associated with this manuscript.

\section{References}

1. Cypess AM, Lehman S, Williams G et al (2009) Identification and importance of brown adipose tissue in adult humans. N Engl J Med 360:1509-1517

2. Saito M, Okamatsu-Ogura Y, Matsushita M et al (2009) High incidence of metabolically active brown adipose tissue in healthy adult humans: effects of cold exposure and adiposity. Diabetes 58:15261531

3. van Marken Lichtenbelt WD, Vanhommerig JW, Smulders NM et al (2009) Cold-activated brown adipose tissue in healthy men. N Engl J Med 360:1500-1508

4. Virtanen KA, Lidell ME, Orava J et al (2009) Functional brown adipose tissue in healthy adults. N Engl J Med 360:1518-1525

5. Zingaretti MC, Crosta F, Vitali A et al (2009) The presence of UCP1 demonstrates that metabolically active adipose tissue in the neck of adult humans truly represents brown adipose tissue. FASEB J 23: 3113-3120

6. Carey AL, Formosa MF, Van Every B et al (2013) Ephedrine activates brown adipose tissue in lean but not obese humans. Diabetologia 56:147-155 
7. Vijgen GH, Bouvy ND, Teule GJ, Brans B, Schrauwen P, van Marken Lichtenbelt WD (2011) Brown adipose tissue in morbidly obese subjects. PLoS One 6:e17247

8. Carey AL, Kingwell BA (2013) Brown adipose tissue in humans: therapeutic potential to combat obesity. Pharmacol Ther 140:26-33

9. Muzik O, Mangner TJ, Granneman JG (2012) Assessment of oxidative metabolism in brown fat using PET imaging. Front Endocrinol 3: 15

10. van Marken Lichtenbelt WD, Schrauwen P (2011) Implications of nonshivering thermogenesis for energy balance regulation in humans. Am J Physiol Regul Integr Comp Physiol 301:R285-R296

11. Blondin DP, Labbe SM, Tingelstad HC et al (2014) Increased brown adipose tissue oxidative capacity in cold-acclimated humans. J Clin Endocrinol Metab 99:E438-E446

12. van der Lans AA, Hoeks J, Brans B et al (2013) Cold acclimation recruits human brown fat and increases nonshivering thermogenesis. J Clin Invest 123:3395-3403

13. Yoneshiro T, Aita S, Matsushita M et al (2013) Recruited brown adipose tissue as an antiobesity agent in humans. $\mathrm{J}$ Clin Invest 123: 3404-3408

14. Yoneshiro T, Saito M (2014) Activation and recruitment of brown adipose tissue as anti-obesity regimens in humans. Ann Med :1-9

15. Lichtenbelt W, Kingma B, van der Lans A, Schellen L (2014) Cold exposure-an approach to increasing energy expenditure in humans. Trends Endocrinol Metab 25:165-167

16. Ravussin Y, Xiao C, Gavrilova O, Reitman ML (2014) Effect of intermittent cold exposure on brown fat activation, obesity, and energy homeostasis in mice. PLoS One 9:e85876

17. Yoo HS, Qiao L, Bosco C et al (2014) Intermittent cold exposure enhances fat accumulation in mice. PLoS One 9:e96432

18. Skarulis MC, Celi FS, Mueller E et al (2010) Thyroid hormone induced brown adipose tissue and amelioration of diabetes in a patient with extreme insulin resistance. J Clin Endocrinol Metab 95:256-262

19. Astrup A, Bulow J, Madsen J, Christensen NJ (1985) Contribution of BAT and skeletal muscle to thermogenesis induced by ephedrine in man. Am J Physiol 248:E507-E515

20. Cypess AM, Chen YC, Sze C et al (2012) Cold but not sympathomimetics activates human brown adipose tissue in vivo. Proc Natl Acad Sci U S A 109:10001-10005

21. Vosselman MJ, van der Lans AA, Brans B et al (2012) Systemic betaadrenergic stimulation of thermogenesis is not accompanied by brown adipose tissue activity in humans. Diabetes 61:3106-3113

22. Cannon B, Nedergaard J (2004) Brown adipose tissue: function and physiological significance. Physiol Rev 84:277-359

23. Collins S, Surwit RS (2001) The beta-adrenergic receptors and the control of adipose tissue metabolism and thermogenesis. Recent Prog Horm Res 56:309-328

24. Young P, Wilson S, Arch JR (1984) Prolonged beta-adrenoceptor stimulation increases the amount of GDP-binding protein in brown adipose tissue mitochondria. Life Sci 34:1111-1117

25. Carey AL, Vorlander C, Reddy-Luthmoodoo M, et al. (2014) Reduced UCP-1 content in in vitro differentiated beige/brite adipocytes derived from preadipocytes of human subcutaneous white adipose tissues in obesity. PLoS One accepted for publication

26. Camera DM, Anderson MJ, Hawley JA, Carey AL (2010) Short-term endurance training does not alter the oxidative capacity of human subcutaneous adipose tissue. Eur J Appl Physiol 109:307-316

27. Ouellet V, Routhier-Labadie A, Bellemare W et al (2011) Outdoor temperature, age, sex, body mass index, and diabetic status determine the prevalence, mass, and glucose-uptake activity of 18F-FDG-detected BAT in humans. J Clin Endocrinol Metab 96:192-199

28. Ouellet V, Labbe SM, Blondin DP et al (2012) Brown adipose tissue oxidative metabolism contributes to energy expenditure during acute cold exposure in humans. J Clin Invest 122:545-552
29. Wu J, Bostrom P, Sparks LM et al (2012) Beige adipocytes are a distinct type of thermogenic fat cell in mouse and human. Cell 150: 366-376

30. Orava J, Nuutila P, Lidell ME et al (2011) Different metabolic responses of human brown adipose tissue to activation by cold and insulin. Cell Metab 14:272-279

31. Cypess AM, White AP, Vernochet C et al (2013) Anatomical localization, gene expression profiling and functional characterization of adult human neck brown fat. Nat Med 19:635-639

32. Jespersen NZ, Larsen TJ, Peijs L et al (2013) A classical brown adipose tissue mRNA signature partly overlaps with brite in the supraclavicular region of adult humans. Cell Metab 17:798-805

33. Sharp LZ, Shinoda K, Ohno H et al (2012) Human BAT possesses molecular signatures that resemble beige/brite cells. PLoS One 7: e49452

34. Torriani M, Zanni MV, Fitch K et al (2013) Increased FDG uptake in association with reduced extremity fat in HIV patients. Antivir Ther $18: 243-248$

35. van der Lans AA, Wierts R, Vosselman MJ, Schrauwen P, Brans B, van Marken Lichtenbelt WD (2014) Cold-activated brown adipose tissue in human adults - methodological issues. Am J Physiol Regul Integr Comp Physiol

36. Lambert GW, Straznicky NE, Lambert EA, Dixon JB, Schlaich MP (2010) Sympathetic nervous activation in obesity and the metabolic syndrome-causes, consequences and therapeutic implications. Pharmacol Ther 126:159-172

37. Dulloo AG, Seydoux J, Girardier L (1991) Peripheral mechanisms of thermogenesis induced by ephedrine and caffeine in brown adipose tissue. Int J Obes 15:317-326

38. Izawa T, Komabayashi T, Suda K, Kunisada Y, Shinoda S, Tsuboi M (1988) Some characteristics of the beta-adrenergic system in rat adipocyte membranes after the chronic administrations of isoproterenol. Res Commun Chem Pathol Pharmacol 60:253-256

39. Harden TK (1983) Agonist-induced desensitization of the betaadrenergic receptor-linked adenylate cyclase. Pharmacol Rev 35:5-32

40. Champigny O, Ricquier D, Blondel O, Mayers RM, Briscoe MG, Holloway BR (1991) Beta 3-adrenergic receptor stimulation restores message and expression of brown-fat mitochondrial uncoupling protein in adult dogs. Proc Natl Acad Sci U S A 88:10774-10777

41. Liu YL, Toubro S, Astrup A, Stock MJ (1995) Contribution of beta 3 adrenoceptor activation to ephedrine-induced thermogenesis in humans. Int J Obes Relat Metab Disord 19:678-685

42. Wheeldon NM, McDevitt DG, Lipworth BJ (1993) Do beta 3adrenoceptors mediate metabolic responses to isoprenaline. Q J Med 86:595-600

43. Schiffelers SL, Blaak EE, Saris WH, van Baak MA (2000) In vivo beta3-adrenergic stimulation of human thermogenesis and lipid use. Clin Pharmacol Ther 67:558-566

44. Murholm M, Isidor MS, Basse AL et al (2013) Retinoic acid has different effects on UCP1 expression in mouse and human adipocytes. BMC Cell Biol 14:41

45. Lafontaine JA, Day RF, Dibrino J et al (2007) Discovery of potent and orally bioavailable heterocycle-based beta3-adrenergic receptor agonists, potential therapeutics for the treatment of obesity. Bioorg Med Chem Lett 17:5245-5250

46. Rohlfs EM, Daniel KW, Premont RT, Kozak LP, Collins S (1995) Regulation of the uncoupling protein gene (Ucp) by beta 1 , beta 2 , and beta 3 -adrenergic receptor subtypes in immortalized brown adipose cell lines. J Biol Chem 270:10723-10732

47. Cannon B, Nedergaard J (2010) Metabolic consequences of the presence or absence of the thermogenic capacity of brown adipose tissue in mice (and probably in humans). Int J Obes 34(Suppl 1):S7-S16

48. Straznicky NE, Lambert GW, McGrane MT et al (2009) Weight loss may reverse blunted sympathetic neural responsiveness to glucose 
ingestion in obese subjects with metabolic syndrome. Diabetes 58: $1126-1132$

49. Straznicky NE, Eikelis N, Nestel PJ et al (2012) Baseline sympathetic nervous system activity predicts dietary weight loss in obese metabolic syndrome subjects. J Clin Endocrinol Metab 97:605-613

50. Sumithran P, Prendergast LA, Delbridge E et al (2011) Long-term persistence of hormonal adaptations to weight loss. N Engl J Med $365: 1597-1604$ 